\title{
MRI points to inflammation as source of cartilage damage
}

$\mathrm{B}$ one erosion, cartilage damage and inflammation-three aspects of the clinical manifestations of rheumatoid arthritis (RA) - interact at a molecular level in the underlying pathology of the disease. Characterizing the sequence of microscopic pathogenic events in RA and relating them chronologically to macroscopic morphological changes is the focus of much ongoing research. How these features interlink is important to understand; submorphological changes occur early in the disease process, are potentially reversible, and might be useful in predicting and preventing irreversible damage. One hurdle in defining interactions of the RA pathological triad, however, is that whereas clinical tools exist to assess inflammation and bone erosion in patients, measuring cartilage degradation presents a greater challenge. Now, Stephanie Finzel and colleagues have used special MRI sequences to visualize biochemical changes in the articular cartilage in the fingers of patients with RA, and compared the results with assessments of inflammation and bone erosion. The study, published in Annals of the Rheumatic Diseases, indicates that the presence of inflammation, and not of bone erosion, correlates with altered composition of cartilage in RA.

"Morphological changes in the cartilage have been shown to be related to decreased physical function in RA," begins Finzel. "Microscopic changes may long precede the morphological changes." Such microscopic changes include alterations in the water content and collagen network of cartilage, which can be measured using special MRI sequences including delayed gadolinium-enhanced MRI of the cartilage (dGEMRIC) and T2 mapping.

Finzel and colleagues used 3-Tesla MRI to scan the metacarpophalangeal joints of 29 patients with RA. "3-T MRI enables visualization of both microanatomical and macroanatomical properties of cartilage
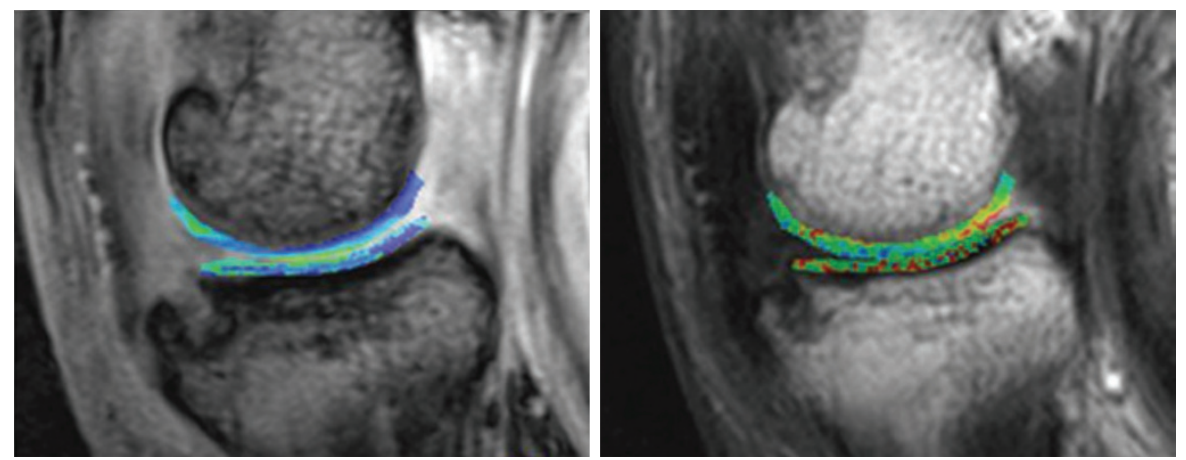

MRI of the $2^{\text {nd }}$ metacarpophalangeal joint of a patient with rheumatoid arthritis showing an erosion. Left-hand panel: a corresponding delayed gadolinium-enhanced MRI of the cartilage map is overlaid; the right-hand panel shows the T2 map. ๑ Herz, B. et al. Ann. Rheum. Dis. doi:10.1136/annrheumdis-2012-202-850 (2013), with permission from BMJ Publishing Group Ltd.

and underlying bone in high resolution;" explains Finzel, "it is more widely available than 7-T MRI and has very good image quality capable of showing even the smallest changes of cartilage and jointspace width." Such sensitivity is crucial for the assessment of small joints such as those of the fingers, in which cartilage width can be $<1 \mathrm{~mm}$. The investigators assayed biochemical properties of the cartilage via dGEMRIC and T2-mapping, and the RA MRI scoring (RAMRIS) system was used to quantify synovitis, osteitis and bone erosions. Macroanatomical parameterscartilage thickness, interbone joint space and intercartilage joint space-were also measured.

"Early changes in the cartilage, corresponding to low dGEMRIC and high $\mathrm{T} 2$ values, were more likely to be seen in joints with decreased interbone joint space and intercartilage joint space as well as decreased cartilage thickness," notes Finzel. "Also osteitis, and to some extent synovitis, correlated with biochemical cartilage changes."

Correlations between inflammation and cartilage microstructure damage were mostly nonsignificant after statisticial adjustment, but low dGEMRIC values in the second metacarpophalangeal joint correlated significantly with the degree of synovitis. By contrast, says Finzel, "late changes in bone (that is, bone erosions) did not correlate with biochemical changes in cartilage."
The findings imply that synovitis and osteitis are the triggers for cartilage damage, and thus that inflammatory burden (and not bone erosions) in a joint determines local cartilage function. Markers of systemic inflammation did not correlate with microanatomical cartilage changes.

Feasibly, microscopic damage to cartilage might be reversible, but whether function recovers in the absence of inflammation is as yet unknown.

Although microscopic cartilage parameters were highly correlated with cartilage thickness and intercartilage joint space at the single timepoint of assessment, the study lacked longitudinal follow-up to investigate how the relationship changed over time. Furthermore, comparisons with other studies are difficult as no standard protocol yet exists for biochemical imaging of cartilage in small joints.

To strengthen the data, the researchers plan a follow-up study in the same patients. They also intend "to scan newly diagnosed patients with RA in another study, to test whether our theories hold true," states Finzel.

Emma Leah

Original article Herz, B. et al. Osteitis and synovitis, but not bone erosion, is associated with proteoglycan loss and microstructure damage in the cartilage of patients with rheumatoid arthritis. Ann. Rheum. Dis. doi:10.1136/ annrheumdis-2012-202850 